\title{
SELEÇÃO DOS INDICADORES DA QUALIDADE DAS ÁGUAS SUPERFICIAIS PELO EMPREGO DA ANÁLISE MULTIVARIADA ${ }^{1}$
}

\author{
EUNICE M. DE ANDRADE ${ }^{2}$, LÚCIA DE F. P. ARAÚJO3 ${ }^{3}$, MORSYLEIDE F. ROSA ${ }^{4}$, \\ WALT DISNEY ${ }^{5}$, AURILEA B. ALVES
}

\begin{abstract}
RESUMO: Os fatores antrópicos e naturais que determinam a qualidade das águas superficiais na Bacia do Baixo Acaraú foram identificados pelo emprego da técnica de Análise Fatorial/Análise da Componente Principal (AF/ACP). Foram realizadas quatro campanhas no período de fev./2003 a mar./2004, em sete pontos de amostragem localizados ao longo do Rio Acaraú. Foram analisados 25 parâmetros físico-químicos e bacteriológicos. Pelo emprego da AF/ACP, identificou-se que o modelo de melhor ajuste para expressar a qualidade das águas na Bacia do Baixo Acaraú foi aquele composto por quatro fatores, explicando $82,1 \%$ da variância total. $\mathrm{O}$ primeiro fator representa a componente de sólidos em suspensão, expressando o processo de erosão e escoamento superficial. O segundo fator é definido por uma componente de nutrientes associados aos esgotos sanitários, às áreas agrícolas e a outras fontes de poluição difusa; o terceiro fator é indicativo da ação antrópica (componente orgânica), enquanto o quarto representa os sais solúveis que expressam o processo natural do intemperismo das rochas. Outro ponto observado é que a análise fatorial não resultou em grande redução no número de variáveis, uma vez que o melhor ajuste do modelo ocorreu com a inclusão de 18 das 25 variáveis analisadas.
\end{abstract}

PALAVRAS-CHAVE: análise fatorial, poluição hídrica, bacia hidrográfica.

\section{SURFACE WATER QUALITY INDICATORS IN LOW ACARAÚ BASIN, CEARÁ, BRAZIL, USING MULTIVARIABLE ANALYSIS}

\begin{abstract}
Multivariate statistical technique, factor analysis/principal component analysis (FA/PCA), was applied to identify the human activity effects and natural processes on surface water quality in Low Acaraú basin, Ceará, Brazil. Water samples were collected in four different campaigns from Feb/2003 to Mar/2004, at seven sampling stations sited in low Acaraú watershed. Twenty-five physical, chemical and bacteriological parameters were examined in this study. The FA/PCA allowed the identification of the total variance. The best model was adjusted with four components, explaining $82.1 \%$ of the data variance. The first factor was mainly associated with suspended solids defining the erosion process and runoff; that is, a suspended solids component. The second factor was mainly associated with sewage, agricultural runoff and others non-pontual pollution; the third one was defined as an organic component and represents the anthropogenic activites; while the fourth was mainly correlated with soluble salt, expressing the weathering and leaching. Besides, the factorial analysis did not result in data reduction, since 18 of the 25 parameters have been kept to explain $82 \%$ of the data variance.
\end{abstract}

KEYWORDS: factor analysis, water pollution, watershed.

\footnotetext{
${ }^{1}$ Pesquisa financiada pelo PRODETAB 16

${ }^{2}$ Eng ${ }^{a}$ Agrônoma, Profa. Ph. D., Departamento de Engenharia Agrícola, CCA/UFC, Caixa Postal 6003, Campus do Pici, Fortaleza CE, Fone: (0XX85) 3366.9762, Fax: (0XX85) 3366.9754, eandrade@ufc.br

${ }^{3}$ Eng $^{\mathrm{a}}$ Química, M.Sc., Professora do CEFET - CE.

${ }^{4}$ Eng ${ }^{\mathrm{a}}$ Química, D.Sc., Pesquisadora da Embrapa Agroindústria Tropical, Fortaleza - CE.

${ }^{5}$ Eng $^{\mathrm{O}}$ Agrícola, Técnico da Companhia de Recursos Hídricos do Estado do Ceará.

${ }^{6}$ Estudante de graduação em Geografia, UECE, Bolsista do PIBIC/CNPq/EMBRAPA.

Recebido pelo Conselho Editorial em: 4-1-2007
}

Aprovado pelo Conselho Editorial em: 12-9-2007 


\section{INTRODUÇÃO}

A qualidade das águas superficiais é facilmente alterada pela adição de efluentes ao sistema de drenagem. O uso dos recursos naturais pelo homem em áreas urbanas, na indústria, em atividades agrícolas, aliado aos processos naturais como variação da precipitação, intemperismo das rochas e erosão alteram a qualidade das águas, tornando-as inadequadas para o consumo humano, recreação, indústria e agricultura (MENDIGUCHÍA et al., 2004; VIDAL et al., 2000).

Os parâmetros físicos, químicos e biológicos que caracterizam a qualidade das águas sofrem grandes variações no tempo e no espaço, havendo a necessidade de um programa de monitoramento sistemático para obter a real estimativa da variação da qualidade das águas superficiais. Em geral, um programa de monitoramento inclui coletas freqüentes nos mesmos pontos de amostragem e análise em laboratório de grande número de parâmetros, resultando em matriz de grandes dimensões e complexa interpretação. Muitas vezes, pequeno número desses parâmetros contém as informações químicas mais relevantes, enquanto a maioria das variáveis adiciona pouco ou nada à interpretação dos resultados em termos de qualidade (SIMEONOV et al., 2003; TOLEDO \& NICOLELLA, 2002).

Os métodos de análise exploratória de dados multivariados são largamente utilizados quando se deseja promover a redução do número de variáveis com o mínimo de perda de informação (HELENA et al., 2000; PALÁCIO, 2004). Na avaliação de dados de monitoramento ambiental, técnicas de estatística multivariada, como Análise Fatorial/Análise da Componente Principal, vêm sendo empregadas com muita frequiência (ANDRADE et al., 2005; MEIRELES et al., 2005). O método das componentes principais é um dos mais usados para resolver problemas clássicos de análise fatorial. Essa análise permite a redução da dimensão de dados, facilitando a extração de informações que serão de grande relevância na avaliação da qualidade das águas e no manejo da bacia.

Com o objetivo de identificar a origem dos fatores que determinam a qualidade das águas na parte baixa da Bacia do Acaraú, aplicou-se o modelo de estatística multivariada - Análise Fatorial/Análise da Componente Principal.

\section{MATERIAL E MÉTODOS}

A área experimental da pesquisa foi a Bacia Hidrográfica do Baixo Acaraú, a qual apresenta área de drenagem de $4.243 \mathrm{~km}^{2}$ e encontra-se localizada entre as coordenadas $2^{\circ} 30^{\prime}$ e $3^{\circ} 45^{\prime} \mathrm{S}$ e $39^{\circ} 30^{\prime}$ e $41^{\circ} \mathrm{W}$. A referida bacia apresenta características bem diferenciadas para cada margem do seu rio principal. A margem direita apresenta relevo e solos característicos da região do sertão, com predominância de substrato cristalino e de clima tipo BSh'w'(semi-árido quente com chuvas de outono). A margem esquerda apresenta-se sob a influência de importantes maciços residuais, como a serra da Meruoca e a encosta da Ibiapaba, as quais promovem chuvas do tipo orográfico. Nessa região, predominam solos de origem sedimentar e clima classificado como Aw' (tropical chuvoso, com chuvas de outono). A média anual da precipitação é de $938 \mathrm{~mm}$ e $80 \%$ desse total ocorre nos meses de março e abril. A evapotranspiração potencial média anual é de $1.600 \mathrm{~mm}$, com temperatura média anual superior a $28{ }^{\circ} \mathrm{C}$ e umidade relativa do ar em torno de $70 \%$.

Para identificar os fatores determinantes da qualidade das águas na Bacia do Baixo Acaraú, realizaram-se coletas trimestrais de água em sete estações amostrais (Figura 1) durante o período de fev./2003 a mar./2004. Os critérios adotados para eleger os pontos de amostragem foram a existência de aglomerados urbanos, de indústrias e de perímetros irrigados. Neste estudo, consideraram-se os seguintes parâmetros: $\mathrm{pH}$, temperatura, cor, turbidez, nitrato, amônia total, amônia livre, amônia ionizada, sódio, cloretos, potássio, sulfato, dureza, cálcio, magnésio, alcalinidade total, alcalinidade do bicarbonato, condutividade elétrica, sólidos suspensos, oxigênio dissolvido (OD), demanda bioquímica de oxigênio $\left(\mathrm{DBO}_{5}\right)$, fósforo total, ortofosfato solúvel, clorofila "A", coliformes totais e coliformes termotolerantes. As análises das amostras de água foram realizadas de acordo com metodologia apresentada por APHA (1998). 


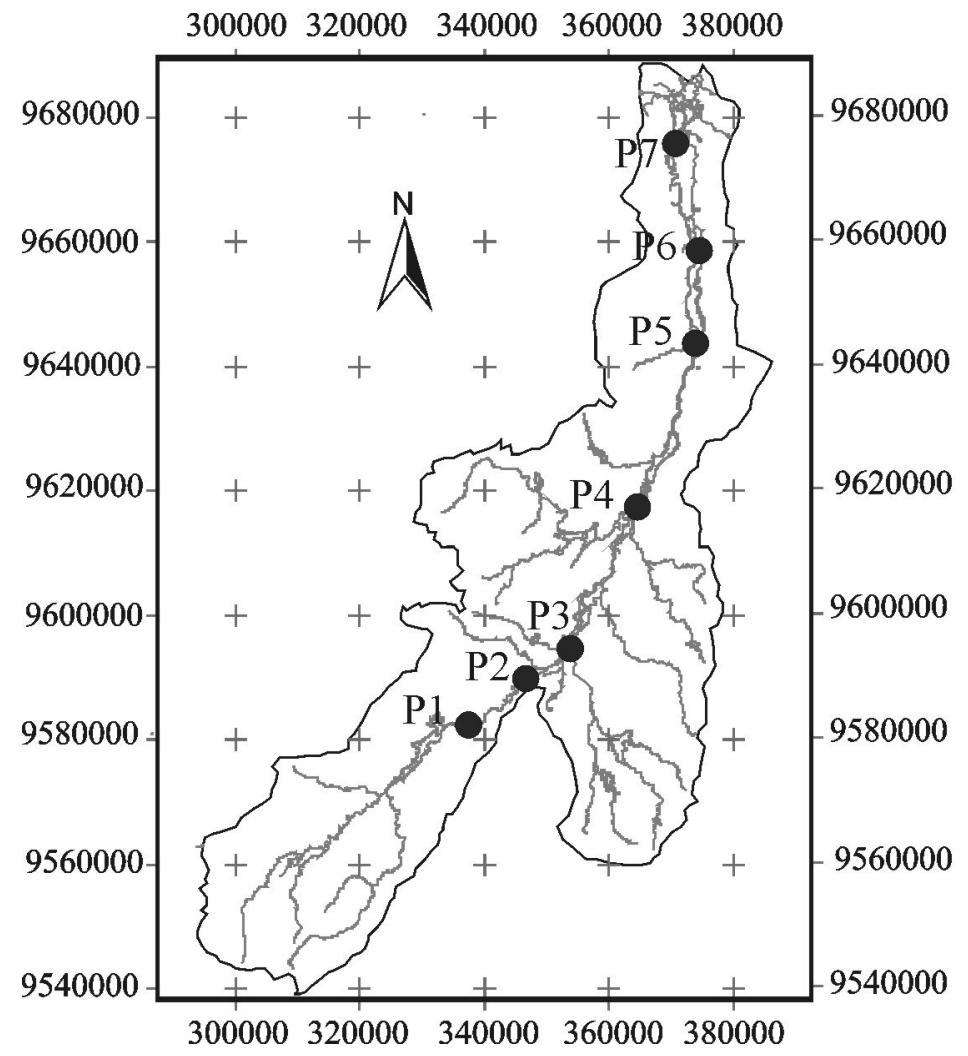

\section{Estações amostrais}

P1- Açude Jaibaras

P2 - Rio Jaibaras

P3 - Rio Madeira

P4 - Açude Acaraú-Mirim

P5 - Rio Acaraú

P6 - Barragem Santa Rosa

P7 - Rio Acaraú, Marco

FIGURA 1. Estações amostrais (Bacia do Baixo Acaraú).

A análise fatorial/análise da componente principal (AF/ACP) foi aplicada aos dados para avaliar as associações entre as variáveis, evidenciando a participação individual dos elementos físicos, químicos e biológicos na qualidade das águas. A AF/ACP consiste em reescrever as variáveis originais em novas variáveis, denominadas componentes principais, por meio de transformação de coordenadas. Esse processo possibilita transformar linearmente um conjunto de variáveis originais em um novo conjunto, menor, de variáveis não-correlacionadas, facilitando o manuseio e a compreensão do fenômeno, sem perda significativa de informação (PALÁCIO, 2004).

Os dados originais, em forma de matriz, eram expressos por $\mathrm{X}=\left(\mathrm{x}_{i, j}\right)$ em que, $i=1 \ldots n$ amostragens (28) e $j=1 \ldots p$ variáveis (25). Na aplicação da técnica da Análise Fatorial/Análise da Componente Principal (AF/ACP), o primeiro passo é transformar a matriz de dados originais em matriz de correlação [R] ( $p$ x $p$ ), para $p$ igual aos 25 parâmetros de qualidade de água considerados nesta pesquisa. Emprega-se a matriz de correlação para eliminar problemas de escalas e unidades diferenciadas em que as variáveis são medidas. A eficiência da descrição dos dados por meio das componentes vai depender da percentagem de variação total que cada componente contém, a qual é representada pelos autovalores (HAIR et al., 2005; VEGA et al., 1998).

O método das componentes principais consiste essencialmente em reescrever as coordenadas das variáveis em outro sistema de eixo mais conveniente para a análise dos dados. Em outras palavras, as n-variáveis originais geram, por meio de suas combinações lineares, n-componentes principais, cuja principal característica, além da ortogonalidade, é que são obtidas em ordem decrescente de máxima variância, ou seja:

$$
\text { VAR } \mathrm{CP}_{1}>\text { VAR } \mathrm{CP}_{2}>\ldots .>\text { VAR } \mathrm{CP}_{\mathrm{n}}
$$

São calculadas de forma que a primeira componente principal explique o máximo da variabilidade total dos dados; a segunda explique o máximo da variabilidade total restante dos dados, sendo não-correlacionada com a primeira; a terceira explique o máximo da variabilidade total restante dos dados, sendo não-correlacionada com a primeira e a segunda componentes, e 
assim sucessivamente até que o número de componentes principais seja no máximo igual ao número de variáveis. Segundo NORUSIS (1990), o modelo matemático para análise de fator apresenta semelhança com uma equação de regressão múltipla, sendo cada variável expressa como uma combinação linear de fatores que não são observados de fato. $O$ modelo para a i-ésima variável normalizada é escrito por meio da regressão linear múltipla entre fatores:

$$
\mathrm{Xsi}=\mathrm{A}_{\mathrm{i} 1} \mathrm{~F}_{1}+\mathrm{A}_{\mathrm{i} 2} \mathrm{~F}_{2}+\ldots+\mathrm{A}_{\mathrm{i} 1} \mathrm{Fl}_{\mathrm{ki}} \ldots+\mathrm{A}_{\mathrm{iL}} \mathrm{F}_{\mathrm{L}}+\xi
$$

em que,

F - fatores comuns, isto é, uma nova variável;

$A_{i}$ - constantes de ajuste do modelo $(i=1, \ldots L)$;

$\xi$ - erro experimental, e

$\mathrm{L}$ - total de fatores.

O modelo assume que os erros experimentais não têm correlação com os fatores comuns (PALÁCIO, 2004).

Todas as análises estatísticas foram formuladas pelo programa SPSS, v.10 (Statistical Package for the Social Sciences) e a seleção do número de componentes extraídos seguiu os preceitos apresentados por NORUSIS (1990), que considera somente componentes com variância superior a um. Esse critério fundamenta-se no fato de que qualquer fator deve explicar uma variância superior àquela apresentada por uma simples variável.

Nem sempre, a matriz das componentes obtidas na fase de extração é de fácil interpretação. Esse fato ocorre quando as cargas dos fatores estão próximas do valor médio. Para suplantar essa limitação, utilizou-se do procedimento de transformação ortogonal, ou simplesmente rotação da matriz das cargas fatoriais, gerando uma nova matriz de cargas fatoriais que apresenta melhor significado interpretativo aos fatores. Neste estudo, empregou-se a rotação ortogonal, método Varimax. Mais informações sobre o assunto podem ser encontradas em DILLON \& GOLDSTEIN (1984); HAIR et al. (2005).

\section{RESULTADOS E DISCUSSÃO}

Das 25 variáveis analisadas, 18 mostraram-se significativas na definição do modelo da análise da componente principal. A extração da matriz de correlação possibilitou a identificação da associação entre as variáveis, as quais podem mostrar a tendência geral dos dados, bem como as variáveis de maior significância para a proposta deste estudo. A correlação entre as 18 variáveis consideradas importantes na definição da qualidade das águas pode ser vista na Tabela 1 . Um total de 72 coeficientes ( $46 \%$ do total) foi superior a 0,5 , o que é indicativo de forte correlação entre as variáveis empregadas (HELENA et al., 2000). Observa-se correlação muito forte e positiva entre as variáveis cor e turbidez $(\mathrm{r}=0,969)$, magnésio e dureza $(\mathrm{r}=0,877)$, cor e sólidos suspensos $(\mathrm{r}=0,876), \mathrm{pH}$ e OD $(\mathrm{r}=0,869)$, sólidos suspensos e DBO $(\mathrm{r}=0,868)$, CE e sódio $(\mathrm{r}=0,847)$, sólidos suspensos e turbidez $(r=0,821)$, sulfato e nitrato $(r=0,818)$, cor e amônia $(r=0,812)$.

As correlações entre cor, turbidez e sólidos suspensos podem ser explicadas pelo fato de que a cor da água é definida pela reflexão e pela refração da luz sobre materiais dissolvidos ou em suspensão. Muitas dessas associações são indicativas de processos não-naturais, como despejo de efluentes sanitários e industriais gerados nas sedes municipais e atividades agropecuárias (VIDAL et al., 2000). A correlação observada entre o magnésio e a dureza era esperada, uma vez que a dureza é definida pela maior ou menor presença de sais dissolvidos de cálcio e magnésio (GASTALDINI \& MENDONÇA, 2001).

A etapa seguinte da análise fatorial constou da decomposição da matriz de correlação para reduzir a dimensão de variáveis interrelacionadas em dimensão menor, formada por fatores comuns e independentes (componente principal). $\mathrm{O}$ modelo que melhor se ajustou aos dados foi aquele composto por quatro componentes, com as raízes características superiores à unidade, e explicando $82,11 \%$ da variância total das variáveis originais (Tabela 2). 
TABELA 1. Matriz de correlação das variáveis fisico-químicas e biológicas significativas do modelo de análise da componente principal.

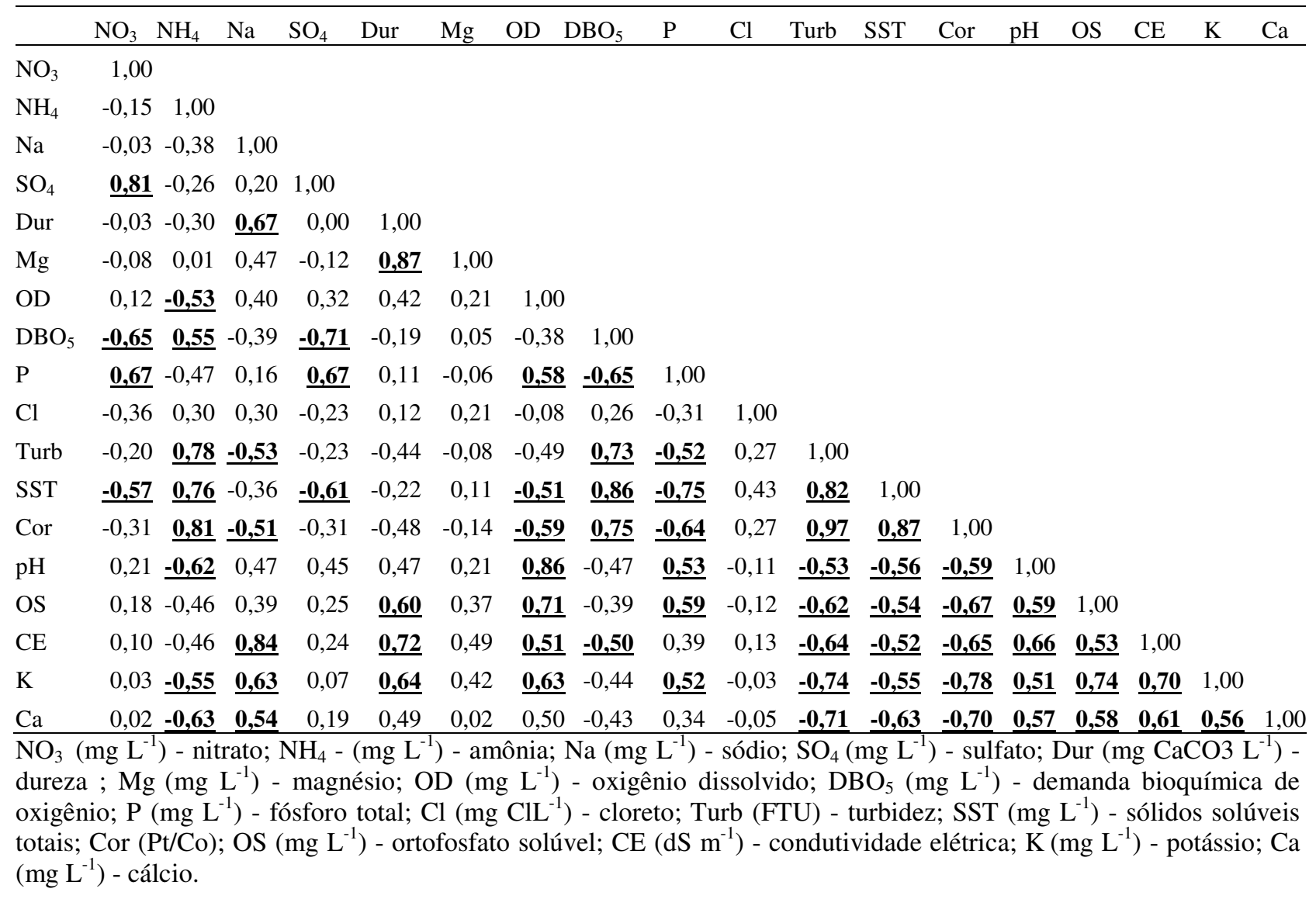

TABELA 2. Fatores das variáveis fisico-químicas e biológicas significativas do modelo de análise da componente principal.

\begin{tabular}{lrrrr}
\hline Variáveis & ACP1 & ACP2 & ACP3 & ACP4 \\
\hline Cor & $\mathbf{- 0 , 9 2 9}$ & 0,057 & 0,245 & 0,077 \\
Turbidez & $\mathbf{- 0 , 8 7 5}$ & 0,021 & 0,352 & 0,171 \\
Sólidos suspensos & $\mathbf{- 0 , 8 6 4}$ & 0,423 & 0,116 & 0,130 \\
Potássio & $\mathbf{0 , 8 0 4}$ & 0,338 & $-0,103$ & 0,110 \\
CE & $\mathbf{0 , 7 8 3}$ & 0,400 & 0,168 & $-0,244$ \\
pH & $\mathbf{0 , 7 7 6}$ & 0,060 & 0,095 & 0,255 \\
Ortofosfato solúvel & $\mathbf{0 , 7 6 9}$ & 0,173 & 0,038 & 0,407 \\
Amônia & $\mathbf{- 0 , 7 6 8}$ & 0,110 & 0,421 & 0,018 \\
DBO & $\mathbf{- 0 , 7 6 4}$ & 0,428 & $-0,131$ & 0,326 \\
OD & $\mathbf{0 , 7 4 2}$ & 0,100 & 0,034 & 0,456 \\
Cálcio & $\mathbf{0 , 7 4 0}$ & 0,151 & $-0,334$ & $-0,154$ \\
Fósforo total & $\mathbf{0 , 7 2 1}$ & $-0,461$ & 0,204 & 0,211 \\
Sódio & $\mathbf{0 , 6 4 3}$ & $\mathbf{0 , 5 0 2}$ & 0,164 & $-0,430$ \\
Magnésio & 0,262 & $\mathbf{0 , 7 0 9}$ & 0,463 & 0,167 \\
Nitrato & 0,382 & $\mathbf{- 0 , 6 8 4}$ & $\mathbf{0 , 5 2 6}$ & $-0,049$ \\
Dureza & 0,598 & $\mathbf{0 , 6 6 3}$ & 0,241 & 0,073 \\
Sulfato & 0,495 & $\mathbf{- 0 , 5 9 8}$ & $\mathbf{0 , 5 2 7}$ & $-0,117$ \\
Cloretos & $-0,208$ & $\mathbf{0 , 5 6 1}$ & 0,251 & $-0,371$ \\
\hline Autovalor & 8,90 & 3,26 & 1,51 & 1,11 \\
\% variância explicada & 49,42 & 18,13 & 8,40 & 6,15 \\
\% variância acumulada & 49,42 & 67,56 & 75,96 & 82,11 \\
\hline
\end{tabular}


A primeira componente explica $49,42 \%$ da variabilidade dos dados e está associada com variáveis indicativas, principalmente das ações antrópica e geológica. Na elaboração de um índice de qualidade de água em uma microbacia de uso agrícola e urbana no município de Guaíra - SP, TOLEDO \& NICOLELLA (2002) também encontraram que a primeira componente abrangeu maior número de variáveis, sendo as de maior peso o fósforo e a amônia. As componentes ACP2 e ACP3 explicaram 18,13 e 8,40\% da variância total dos dados originais, respectivamente. Pode-se observar que ambas apresentaram variáveis comuns (nitrato e potássio) na explicabilidade da variabilidade, enquanto a ACP4 não expressou nenhuma variável com peso significativo, tornando a matriz de fatores de difícil interpretação. Para superar essa limitação, aplicou-se a transformação ortogonal ou simplesmente rotação dos eixos. O processo maximiza a variância entre os fatores, alterando a raiz característica sem afetar a proporção da variância total explicada pelo conjunto. Esse procedimento vem sendo empregado por pesquisadores, como HELENA et al. (2000), SILVEIRA \& ANDRADE (2002), MENDIGUCHÍA et al. (2004) e PALÁCIO (2004) em estudos de qualidade da água com a finalidade de minimizar a contribuição das variáveis com menor significância no fator. Os novos pesos atribuídos a cada variável, bem como a variância total explicada por cada componente é apresentada na Tabela 3. As componentes da matriz rotacionada pelo método varimax registraram melhor distribuição da variância original. A primeira componente rotacionada (VF1) passou a explicar $29,45 \%$ da variância, enquanto a terceira componente rotacionada (VF3), bem como a quarta componente rotacionada (VF4) passaram a contribuir com $17,91 \%$ e $15,59 \%$ em substituição aos $8,40 \%$ e $6,15 \%$ registrados antes da transformação ortogonal, respectivamente. Melhor distribuição da variância total entre as componentes pela rotação da matriz também foi observada por HELENA et al. (2000).

TABELA 3. Fatores das componentes rotacionadas (VF) das variáveis fisico-químicas e biológicas significativas do modelo de analise da componente principal.

\begin{tabular}{lcccc}
\hline Variáveis & VF1 & VF2 & VF3 & VF4 \\
\hline Turbidez & $\mathbf{0 , 9 0 2}$ & $-0,176$ & $-0,255$ & $-0,103$ \\
Cor & $\mathbf{0 , 8 4 6}$ & $-0,271$ & $-0,364$ & $-0,104$ \\
Amônia & $\mathbf{0 , 8 1 7}$ & $-0,125$ & $-0,298$ & 0,087 \\
Cálcio & $\mathbf{- 0 , 7 8 1}$ & 0,017 & 0,241 & 0,193 \\
Sólidos suspensos & $\mathbf{0 , 7 5 3}$ & $-0,576$ & $-0,224$ & 0,087 \\
Potássio & $\mathbf{- 0 , 6 0 0}$ & 0,016 & 0,560 & 0,347 \\
Nitrato & $-0,001$ & $\mathbf{0 , 9 3 6}$ & 0,069 & $-0,110$ \\
Sulfato & $-0,102$ & $\mathbf{0 , 9 3 6}$ & 0,089 & 0,008 \\
DBO & $-0,591$ & $\mathbf{- 0 , 7 2 2}$ & $-0,042$ & $-0,089$ \\
Fósforo Total & $-0,342$ & $\mathbf{0 , 6 8 4}$ & 0,464 & $-0,135$ \\
OD & $-0,355$ & 0,178 & $\mathbf{0 , 7 7 8}$ & 0,083 \\
Ortofosfato Solúvel & $-0,385$ & 0,150 & $\mathbf{0 , 7 6 8}$ & 0,164 \\
pH & $-0,412$ & 0,288 & $\mathbf{0 , 6 2 9}$ & 0,178 \\
Sódio & $-0,493$ & 0,095 & 0,104 & $\mathbf{0 , 7 8 5}$ \\
Magnésio & 0,173 & $-0,108$ & 0,484 & $\mathbf{0 , 7 3 2}$ \\
Dureza & $-0,236$ & $-0,064$ & 0,540 & $\mathbf{0 , 7 1 4}$ \\
CE & $-0,531$ & 0,191 & 0,306 & $\mathbf{0 , 6 7 0}$ \\
Cloretos & 0,195 & $-0,241$ & $-0,256$ & $\mathbf{0 , 6 3 0}$ \\
\hline Autovalor & 5,30 & 3,45 & 3,22 & 2,81 \\
\% variância explicada & 29,45 & 19,17 & 17,91 & 15,59 \\
\% variância acumulada & 29,45 & 48,62 & 66,53 & 82,11 \\
\hline
\end{tabular}

A VF1 apresentou elevados coeficientes positivos para a turbidez, cor, amônia e sólidos suspensos, e coeficientes negativos para o cálcio e o potássio. Basicamente, representa uma componente de sólidos. Essa maior carga de sólidos, possivelmente, tem sua origem no escoamento superficial das áreas agrícolas e nos depósitos de resíduos sólidos existentes próximos aos cursos 
d'água. A participação do cálcio e do potássio no fator que detém o maior percentual da variância total pode ser explicada pela presença de rochas hornblendas, ricas em cálcio e potássio, na formação dos solos da Bacia do Acaraú.

A VF2 (segunda componente rotacionada) apresentou coeficientes elevados e positivos $(>0,935)$ com o nitrato e o sulfato, e valores de coeficientes moderados com a DBO e o fósforo. Essa componente "nutricional" expressa a influência da poluição difusa, tais como o escoamento superficial das áreas agrícolas e o uso extensivo dos fertilizantes nitrogenados nos perímetros irrigados que ficam localizados na parte baixa da Bacia do Acaraú.

Alterações na qualidade das águas superficiais devidas ao escoamento superficial das áreas agrícolas e ao emprego de fertilizantes nitrogenados foram registradas por SIMEONOV et al. (2003), durante a avaliação da qualidade das águas superficiais no nordeste da Grécia. Outra fonte de poluição que pode contribuir para esse grupo é o esgoto doméstico lançado nos cursos d'água ao longo de toda a Bacia. Dados do IBGE (2002) revelam que, em 2000, a forma de esgotamento sanitário de 49,12\% dos domicílios particulares das áreas urbanas da Bacia do Acaraú eram do tipo fossa rudimentar, vala negra, rio, lago, mar ou outro tipo de escoadouro, o que contribui fortemente para o aumento de fontes poluidoras ao longo da rede de drenagem da Bacia do Baixo Acaraú.

A VF3 recebeu maior peso do $\mathrm{OD}$, ortofosfato solúvel e $\mathrm{pH}$, sendo componente indicadora da poluição orgânica. Essa VF apresentou-se como componente indicativa da ação antrópica na qualidade das águas.

Em estudos sobre as águas no Nordeste da Grécia, SIMEONOV et al. (2003) encontraram uma terceira componente semelhante à observada nesta pesquisa, sendo considerada como indicativo da variabilidade físico-química das águas estudadas. Os elementos que apresentaram maior correlação com a VF4 foram sódio, magnésio, dureza, CE e cloreto, a qual pode ser interpretada como componente mineral da água do Rio Acaraú. Esse grupo de variáveis aponta para uma origem comum desses minerais, tais como: intemperismo das rochas que deram origem ao solo e conseqüente escoamento superficial das áreas drenadas pelo Rio Acaraú. Resultados semelhantes foram encontrados por MEIRELES et al. (2005) trabalhando com parâmetros indicadores da qualidade das águas de reservatórios no Ceará, onde encontraram a CE e o sódio como os elementos de maior peso da segunda ACP. Outro ponto a ser considerado é o largo emprego do calcário dolomítico para corrigir a acidez do solo nas áreas do Distrito de Irrigação do Baixo Acaraú, o qual apresenta pH menor que 5 (ANDRADE, 2004). Acredita-se que os cloretos encontrados nas águas do Rio Acaraú sejam em decorrência da lavagem de roupas e de despejos de esgotos domésticos dispostos no rio, uma vez que o cloreto não é mineral predominante nos solos da bacia (DNOCS, 1977). Em estudos na bacia do Rio Trussu, PALÁCIO (2004) identificou como fonte de cloreto as lavagens de roupa e os esgotos domésticos.

\section{CONCLUSÕES}

A técnica da Análise Fatorial/Análise da Componente Principal permitiu a seleção de quatro componentes indicadoras da qualidade das águas superficiais, explicando 82,1\% da variância total. As variações da qualidade da água foram definidas por um grupo de sólidos (representando o processo de erosão e escoamento superficial), por um grupo de nutrientes (influência de fontes de poluição difusa, como drenagem das áreas agrícolas), por um grupo orgânico (indicativo da ação antrópica) e um grupo de sais solúveis (processo natural).

A análise fatorial não promoveu grande redução no número de variáveis, uma vez que o melhor ajuste do modelo ocorreu com a inclusão de 18 das 25 variáveis analisadas.

\section{REFERÊNCIAS}

ANDRADE, E.M.; PALÁCIO, H.A.Q.; CRISÓSTOMO, L.A.; SOUZA, I.H.; TEIXEIRA, A.S. Índice de qualidade de água, uma proposta para o vale do rio Trussu, Ceará. Revista Ciência Agronômica, Fortaleza, v.36, n.2, p.135-42, 2005. 
ANDRADE, E.M. Estudo do risco de degradação dos recursos naturais devido ao manejo de irrigação em bacias hidrográficas na região da caatinga: subprojeto-03. Fortaleza: PRODETAB, 2004. 20 p. (Relatório anual, 16)

APHA. American Public Health Association, Standard Methods for the Examination of Water and Wastewater. $20^{\text {th }}$ ed. Washington, 1998. 1.220p.

DILLON, W.R.; GOLDSTEIN, M. Multivariate analysis methodos and applications. New York: John Wiley, 1984. 587 p.

DNOCS. Plano diretor do Vale do Acaraú. Pedologia, 1977. v.2, 96 p.

GASTALDINI, M.C.C.; MENDONÇA, A.S.F. Conceitos para a avaliação da qualidade da água. In: PAIVA, J.B.D.; PAIVA, E.M.C.D. Hidrologia aplicada à gestão de pequenas bacias hidrográficas. Porto Alegre: ABRH, 2001. cap. 15, p.428-51.

HELENA, B.; PARDO, R.; VEGA, M.; BARRADO, E.; FERNANDEZ J.M.; FERNANDEZ, L. Temporal evolution of groundwater composition in an alluvial aquifer (Pisuerga river, Spain) by principal component analysis. Water Research, Amsterdam, v.34, n.3, p.807-16, 2000.

HAIR, J.J.F.; ANDERSON, R.E.; TATHAM, R.L.; BLACK, W.C. Análise multivariada de dados. 5.ed. Porto Alegre: Bookman, 2005. 593 p.

IBGE. Instituto Brasileiro de Geografia e Estatística. Indicadores de desenvolvimento sustentável: Brasil 2002. Rio de Janeiro, 2002. 195 p.

MEIRELES, A.C.M.; ANDRADE, E.M.; FRISCHKORN, H.; CHAVES, L.C.G.; CRISÓSTOMO, L.A. Identificação dos principais constituintes da salinidade da água no rio Acaraú. In:

CONGRESSO BRASILEIRO DE RECURSOS HIDRICOS, 16., 2005, João Pessoa. Anais... João Pessoa: ABRH. 1 CD-ROM.

MENDIGUCHÍA, C.; MORENO, C.; GALINDO-RIANO, M.D.; GARCÍA-VARGAS, M. Using chemometric tools to assess antropogenic effects in river water a case study: Guadalquivir river (Spain). Analytica Chimica Acta, Amsterdam, v.515, p.143-9, april, 2004.

NORUSIS, M.J. SPSS Base System User's Guide. Chicago: SPSS, 1990. 520 p.

PALÁCIO, H.A.Q. Índice de qualidade das águas na parte baixa da bacia hidrográfica do rio Trussu, Ceará. 2004. 96 f. Dissertação (Mestrado em Irrigação e Drenagem) - Universidade Federal do Ceará, Fortaleza, 2004.

SILVEIRA, S.S.; ANDRADE, E.M. Análise de componente principal na investigação da estrutura multivariada da evapotranspiração. Engenharia Agrícola, Jaboticabal, v.22, n.2, p.171-7, 2002.

SIMEONOV, V.; STRATIS, J.A.; SAMARA, C.; ZACHARIADIS, G.; VOUTSA, D.; ANTHEMIDIS, A.; SOFONIOU, M.; KOUIMTZIS, T. Assessment of the surface water quality in northen Greece. Water Research, Amsterdam, v.37, n.17, p.4.119-24, 2003.

TOLEDO, L.G.; NICOLELLA, G. Índice de qualidade de água em microbacia sob uso agrícola e urbano. Scientia Agrícola, Piracicaba, v.59, n.1, p.181-6, 2002.

VIDAL, M.; LÓPEZ, A.; SANTOALLA, M.C.; VALLES, V. Factor analysis for the study of water resources contamination due to the use of livestock slurries as fertilizer. Agricultural Water Management, Netherlands, v.45, n.1, p.1-15, 2000.

VEGA, M.; PARDO, R.; BARRADO, E.; DEBAN, L. Assessement of seasonal and polluting effects on the quality of river water by exploratory data analysis. Water Research, Amsterdam, v.32, n.12, p.3.581-92, 1998. 